\title{
FLOWER POLLINATION ALGORITHM FOR MULTI LEVEL LOT SIZING OPTIMIZATION
}

\author{
V.V.D.Sahithi ${ }^{1}$, M. Srinivasa Rao $^{2}$, C.S.P.Rao ${ }^{3}$ \\ ${ }^{1}$ Research Scholar, JNTU Hyderabad, India \\ ${ }^{2}$ Professor, JNTU Hyderabad, India \\ ${ }^{3}$ Director, NIT, Warangal, India \\ ${ }^{1}$ sahithicodes@gmail.com, ${ }^{2}$ raoms63@jntuh.ac.in, ${ }^{3}$ csp_rao@rediffmail.com
}

\begin{abstract}
In this competitive and constantly changing world, meeting the customer requirements within less time by providing less cost is extremely tricky task. This is only possible by optimizing all the different parameters in its life cycle. Here Optimizing the inventory plays a major role.Maintaining the exact amount of inventory, at proper place, in appropriate level is a challenging task for production managers. When we work on Multi level environments this problem becomes even more complex.So, to optimize this kind of problems we applied binary form of Flower Pollination algorithm to solve this complex problem. we solved different inventory lot sizing problems with this FP algorithm and compared the results with genetic algorithm and other algorithms. In all the scenarios our simulation results shown that FP algorithm is better than other algorithms.
\end{abstract}

\section{Introduction}

Proper management of inventory is essential for any industry. It acts as the link between production and distribution. If it is not maintained properly it will become a white elephant. If inventory costs increase it shows the direct reflection in product pricing. Hence to optimize the cost basic MRP is not sufficient because its target is to only provide the right number of items to full fill the production requirements in the stipulated time without any deficiency. But in this competitive era with the items having complex product structures cost plays a very important role. One needs to produce the product with less cost to survive in this high competition. So we need to come up with a lot sizing plan which not only satisfies the basic MRP requirement, but also gives us the optimum cost .Here inventory Lot sizing plays a vital role in optimizing the total variable cost which is the sum of holding cost (HC) of inventory and ordering cost (OC) of inventory.

Optimal heuristic and meta heuristic algorithm procedures exists for this problem, but most of them were proven to reach the optimum only in case of single level problems. In ML problems most of these techniques can solve the problem in reasonable time only in case of small instances. But when we work on ML environment with a greater number of levels and multiple sub items, long planning horizons the problem becomes very complex. When we work on real of industrial problems where there are thousands of items there optimizing the cost in reasonable computational time is a really challenging task.

So many heuristics techniques like were used to solve these ML problems .In those one technique is by modifying economic order quantity technique of single level to all the levels of the product structure[1] .The other technique part period balancing defined is by using look head and back strategy[2].one more technique called silver meal heuristic was developed to solve these ML problems by calculating the average costs per period,not only these techniques many other techniques were developed to solve this ML problem. But later researchers started using meta heuristic evolutionary techniques to solve these complex NP hard ML problems[3]. because of their efficient optimization strategies and reasonable computation time these meta heuristic evolutionary techniques took precedence over any other technique.

In early 2000s researchers used genetic algorithms to solve these ML lot sizing issues and gave very good results in terms of solution values for small size problems with limited number of sub items. Later with the success of GA technique many other evolutionary techniques like ant colony (AC), tabu search (TS), simulatedannealing(SA)etc. were developed for theses NP hard problems[4-10]. In recent literature harmonic search (HS) algorithm,Invasive weed (IW) techniques were developed for solving these problems[. Most of these recent techniques were successful in implementation of complex ML, NP hard problems[10-18].But still there is a lot scope and requirement in find the optimal solution in with high computational efficiency.

Here in this research paper our we presented a binary flower pollination algorithm to solve ML assembly structure. This algorithm is tested for feasibility, effectiveness, and computational efficiency. As this algorithm provided the best results, we planned to extend this work to a real-life bus 
manufacturing application which is having thousands of sub items and many BOM levels.

This paper is organized as follows: section2 is about presentation of mathematical formulation of ML lot sizing problem.Section 3 is to present binary FP algorithm encoding, framework and pseudo code. Section 4 is about experimental works and resultsand finally section 5 is conclusion.

\section{Mathematical Formulation:}

In production systems main/final product made up of different sub parts which are raw materials or purchased parts .To satisfy the final product external demand which is generated from the customer requirements we have to make sure that all the sub components are available possibly at lowcost.

BOM generally represents, different components information of an assembly structure. In the Acyclic representation of BOM ,nodes gives the information about the item/subitem.Edge $(\mathrm{k}, \mathrm{r})$ between nodes $\mathrm{k}, \mathrm{r}$ exist when item $\mathrm{k}$ is required to assemble r.Generally $\Gamma^{-1}(\mathrm{k}), \Gamma(\mathrm{k})$ representation is used to represent immediate predecessors \&immediatesuccessors.In BOM structure final component is always at the lowest level.If the levels are from 1 to $n, n$ is mostly a purchased part.

In the mathematical formulation of ML lot sizing problem, we have to find out the final lot sizes of final product as well as all sub parts. So there are different objectives and constraints that needs to be considered while formulating such problem. Following are the considerations to formulate the problem

- No backordering is allowed.

- Components are not allowed to sell outside. So, independent demand exists only for final product .Remaining all items are having dependent demands.

- Lead times are assumed as zero.

- No schedule receipts, no initial inventories.so net and gross requirements are same.

- No variable cost /purchase parameters were taken into consideration.

Table 1

ML lot sizing problem notations

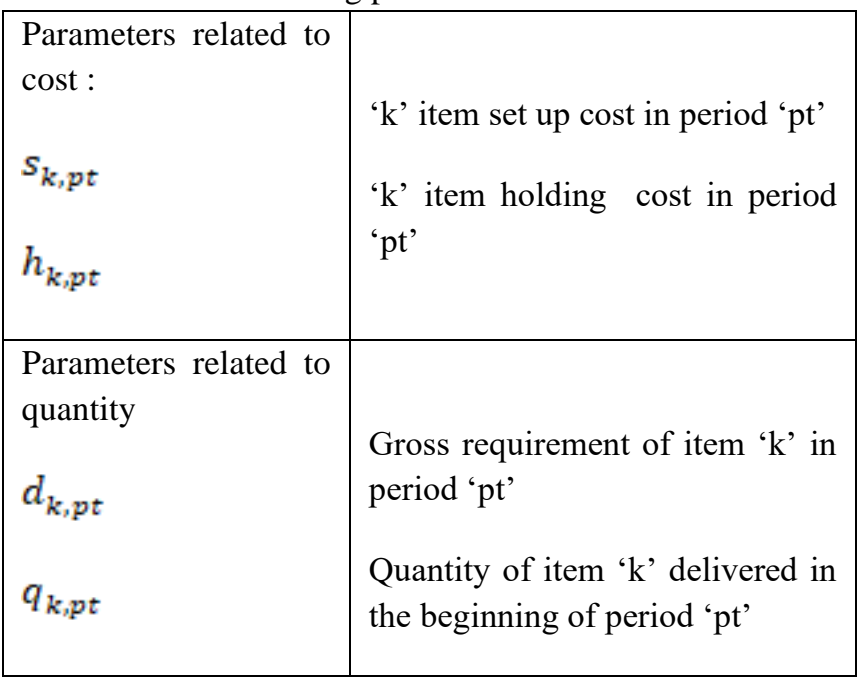

\begin{tabular}{|l|l|}
\hline $\mathrm{I}_{k_{2} p t}$ & $\begin{array}{l}\text { Inventory level of item ' } \mathrm{k} \text { ' } \\
\text { required at the end of period 'pt' } \\
\text { Boolean variable to take decision } \\
\text { wheather order is placed or not }\end{array}$ \\
$b_{k_{2} p t}$ & $\begin{array}{l}\text { Lead time for item } \mathrm{k} \\
\text { Technical } \\
\text { information } \\
l_{k}\end{array}$ \\
$c_{k_{2} r}$ & produce unit of item r $\mathrm{k}$ \\
\hline
\end{tabular}

For problem formulation we followed the references [1] and then modified according to our ML assembly structure requirement.

$$
\begin{aligned}
& \text { Minimize } \sum_{k=1}^{n} \sum_{p t=1}^{T} s_{k_{s} p t} b_{k, p t}+h_{k_{i} p t} I_{k_{i} p t} \\
& I_{k p t}=I_{k p t-1}+q_{k p t}-d_{k p t} \\
& d_{i_{\imath} k t}=\sum_{r \in \Gamma(k)}^{d_{i_{i} k t}} q_{r i p t+l r} c_{k, r} \\
& q_{k p t}-M b_{k p t} \leq 0 \quad b_{k p t} \epsilon\{0,1\} \\
& \mathbf{I}_{k, p t} \geq \mathbf{0} \\
& q_{k_{i p t}} \geq \mathbf{0}
\end{aligned}
$$

The Objective function shown in equation (1) is the minimization of sum of $\mathrm{HC}$ and SC of all the items in the given planning horizon.Equation(2) represents the inventory level at the end of given period.Equation(3) make sure that no items is sold outside customer .Equation(4) tells about the setup cost is applied when a lot is purchased.Equation(5) tells that backlogging of inventory is not allowed \&(6) make sure that production is always positive $/ 0$.

\section{Flower pollination algorithm:}

Pollination is a fascinating phenomenon in the nature. Inspired from its evolutionary characteristics FPA algorithm was developed in 2012 by Xin-She Yang.This algorithm characteristics are defined from the inspiration of reproduction in flowering plants.The biological reproduction process 
characteristics are defined in FPA algorithms by following 4 governing rules[19]

1.

Biotic pollination \& cross pollination was done by the biotic pollinators like insects /birds performing lévyflights.

2.

Abiotic /self pollination uses abiotic non-living methods such as wind to carryout pollination which is called as local pollination.

3. reproduction probability is proportional to the similarity of two flowers involved.

4.

Global or local pollination can be controlled by probability parameter $\mathrm{p}$.

In our algorithm we are mapping the natural process of pollination to FPA. In reality each flower contains millions of pollen grains. But for the simplification purpose we assume each flower is containing one pollen gamete. So one solution typically represents plant/flower/pollen gamete.

FPA algorithm mainly contains 2 steps, in that one is replated to global pollination and the other is related to local pollination .

Global pollination of flowering plant with respect to the algorithm was represented as follows.

$x_{i}^{t+1}=x_{i}^{t}+L\left(x_{i}^{t}-g b\right)$

Here $g b$ represents global best and ${ }^{x_{i}^{t}}$ represents solution vector $^{x_{\tilde{i}}}$ in iteration ' $\mathrm{t}$ '. Here ${ }^{x_{i}^{t+1}}$ is modified ${ }^{x_{\tilde{i}}}$ in the next iteration ' $t+1$ '. Here $\mathrm{L}$ represents insects pollination strength.Insects move different lengths by carrying pollen gametes, which is controlled by Lévy flights taken from lévy distribution.

$$
\begin{aligned}
& \mathrm{L}=\frac{\lambda \Gamma(\lambda) \sin \left(\frac{\lambda \Pi}{2}\right)}{\prod} \frac{1}{s^{1+\lambda}}\left(\mathrm{s}>>\mathrm{s}_{0}>0\right) \\
& \quad s=\frac{U}{|V|^{1 / \beta}}, U \sim N\left(0, \sigma^{2}\right) \text { and } \quad V \sim N(0,1) .
\end{aligned}
$$

Here constant $\lambda$ is used to create lévynumbers.andhere $s$ represents step length and this algorithm is for large step lengths.

Local pollination of the flowers are represented by the following equation

$x_{i}^{t+1}=x_{i}^{t}+\epsilon\left(x_{j}^{t}-x_{k}^{t}\right)$

Here ${ }^{x_{j}^{t}}$ and $x_{k}^{t}$ are the pollen grains from different flowers /solutions in the neighbourhood of solution i. Here $\epsilon$ represents the local walk of abiotic pollination, which is calculated from $[0,1]$ uniform distribution.

Here to convert the solution values to the binary form sigmoid function is used

$$
\begin{aligned}
& \mathrm{S}\left(x_{i}^{p}\right)_{=1+e^{x_{i}^{p}}} \\
& x_{i}^{t}=\left\{\begin{array}{cc}
1 & \mathrm{~S}\left(x_{i}^{p}\right)>\sigma \\
0 & \text { otherwise }
\end{array}\right.
\end{aligned}
$$

Here $\boldsymbol{\sigma}$ value comes from uniform distribution $\mathrm{U}[0,1]$.

\section{Experiments and their simulation results :}

Here we have presented various example problems from the literature to test our FPA algorithm behaviour. To conduct all these simulation experiments authors used MATLAB software.PC specifications are 8GB ram and $2.8 \mathrm{GHZ}$ CPU.All the simulations were performed for 100 times to findout the results

Here 3 different product structures were used with7 different planning horizons.Following are the different problems considered for the Experiments

$1.7 \times 6$ problem [20] represents problem with 6 items BOM structure with 6 periods planning horizon.

2. $6 \times 10,6 \times 12,6 \times 15$ problems [21] here 6 items problem with different planning horizons were considered to compare the simulation results.

$3.9 \times 10,9 \times 12,9 \times 15$ problems [21] here 9 items problem with different planning horizons were considered to compare the simulation results.

In all the cases FPA was applied and and simulation results of

Here $U \sim N\left(0, \sigma^{2}\right)$ denotes that the samples have boptimum value and computational time were compared with drawn from a Gaussian normal distribution with variander algorithms.

of $\sigma^{2}$ and zero mean. For the purpose of calculationTbe following are the simulation results of $7 \times 6$ problem and variance one can use the formula:

$$
\sigma^{2}=\left\{\frac{\lceil(1+\beta)}{\beta\left\lceil\frac{1+\beta}{2}\right.} \frac{\sin \left(\frac{\pi \beta}{2}\right)}{2^{\frac{\beta-1}{2}}}\right\}^{1 / \beta} .
$$
the figure clearly shows the dominance of the FPA algorithm over other evolutionary algorithms like GA ,SA,TS,LR.

\begin{tabular}{|l|l|l|l|}
\hline Size of the Problem & Algorithm type & Optimum cost value & $\begin{array}{l}\text { Simulation time to reach } \\
\text { optimum(sec) }\end{array}$ \\
\hline & FPA & 8320 & $<0.2$ \\
\hline
\end{tabular}




\begin{tabular}{|l|l|l|l|}
\hline \multirow{3}{*}{ 7items $\times 6$ periods } & GA & 9245 & 10.10 \\
\cline { 2 - 4 } & SA & 10740 & 9.90 \\
\cline { 2 - 4 } & TS & 9620 & 8.80 \\
\cline { 2 - 4 } & LR & 9239 & 34.10 \\
\hline
\end{tabular}

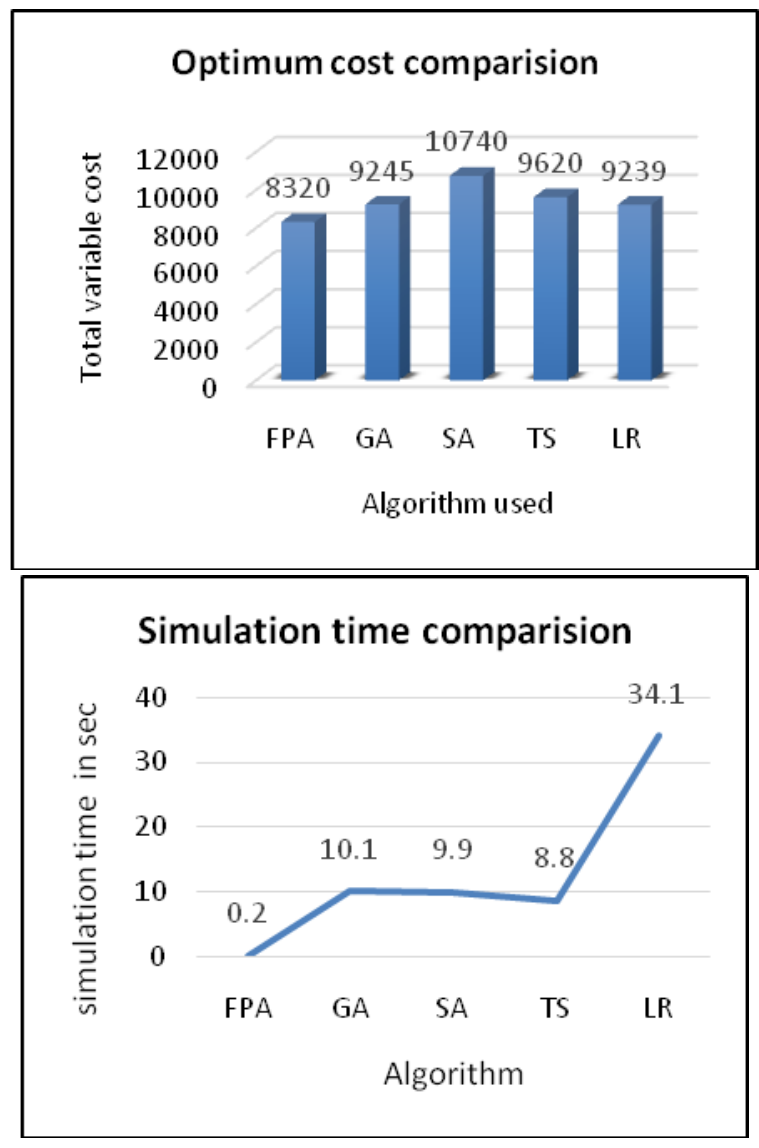

dominance of the FPA algorithm over other evolutionary

The following are the simulation results of $6 \times 10,6 \times 12,6 \times 15$ algorithms like PSO,GA,WW.

problems and the following figure clearly shows the

Table 8

Computational Results of MLLSOP with 6 items and different planning horizons

\begin{tabular}{|l|l|l|l|}
\hline Size of Problem & Algorithm & Optimum cost & Time to find out Optimum solution(s) \\
\hline \multirow{5}{*}{ 6items $\times 10$ periods } & FPA & 1493 & 0.4 \\
\cline { 2 - 5 } & PSO & 1493 & 4.2 \\
\cline { 2 - 5 } & GA & 1493 & 5.6 \\
\cline { 2 - 5 } & WW & 1707 & $<0.1$ \\
\hline \multirow{3}{*}{6 items $\times 12$ periods } & FPA & 1895 & 3.5 \\
\cline { 2 - 5 } & PSO & 1895 & 5.3 \\
\cline { 2 - 4 } & GA & 1895 & 8.0 \\
\hline
\end{tabular}




\begin{tabular}{|l|l|l|l|}
\hline & WW & 2123 & $<0.1$ \\
\hline \multirow{5}{*}{ 6items $\times 15$ periods } & FPA & 2546 & 5.3 \\
\cline { 2 - 4 } & PSO & 2546 & 6.3 \\
\cline { 2 - 4 } & GA & 2623 & 10.7 \\
\cline { 2 - 4 } & WW & 2909 & $<0.1$ \\
\hline
\end{tabular}

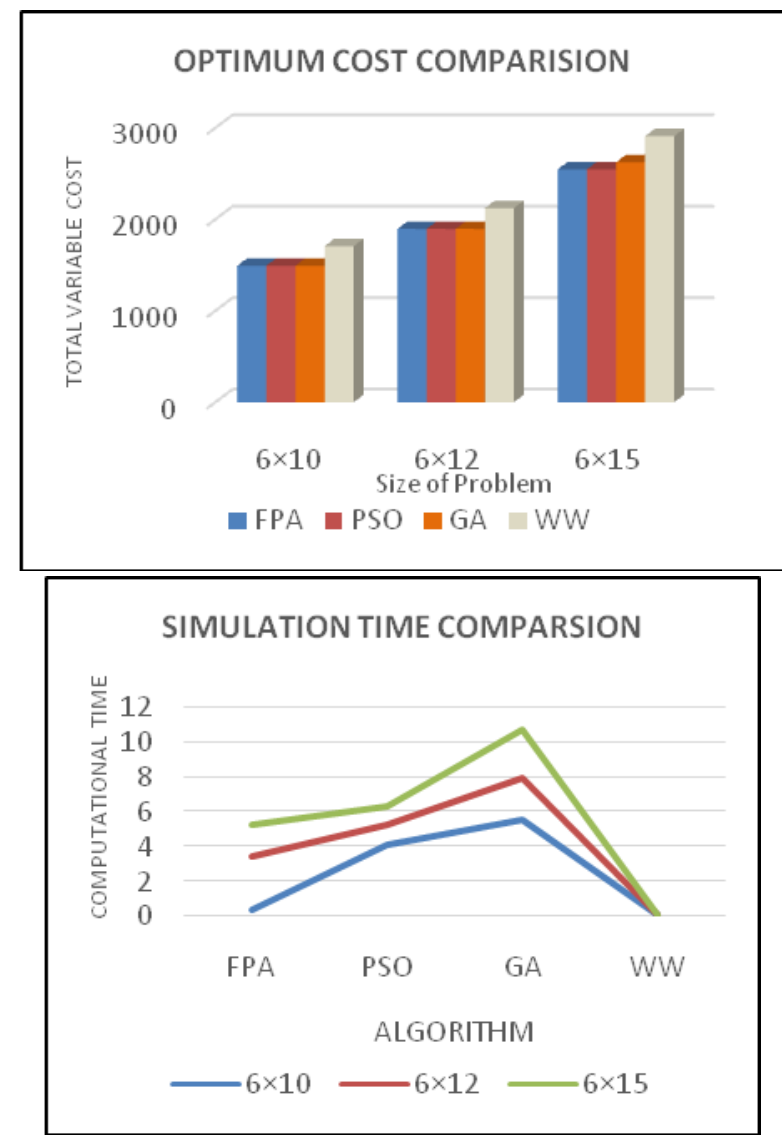

The following are the simulation results of $9 \times 10,9 \times 12,9 \times 15$ dominance of the FPA algorithm over other evolutionary problems and the following figure clearly shows the algorithms like PSO,GA,WW.

\begin{tabular}{|l|l|l|l|}
\hline Size of Problem & Algorithm & Optimum cost & Time to find out Optimum solution(s) \\
\hline \multirow{4}{*}{ 9items $\times 10$ periods } & FPA & 2043 & 4.1 \\
\cline { 2 - 4 } & PSO & 2043 & 6.5 \\
\cline { 2 - 4 } & GA & 2043 & 10.1 \\
\cline { 2 - 4 } & WW & 2807 & $<0.1$ \\
\hline \multirow{5}{*}{ 9items $\times 12$ periods } & FPA & 2522 & 5.7 \\
\cline { 2 - 4 } & GSO & 2522 & 7.2 \\
\cline { 2 - 4 } & GA & 2522 & 12.9 \\
\hline
\end{tabular}




\begin{tabular}{|l|l|l|l|}
\hline & WW & 3498 & $<0.1$ \\
\hline \multirow{4}{*}{9 9items $\times 15$ periods } & FPA & 3448 & 8.5 \\
\cline { 2 - 4 } & PSO & 3448 & 9.2 \\
\cline { 2 - 4 } & GA & 3714 & 16.3 \\
\cline { 2 - 4 } & WW & 4834 & $<0.1$ \\
\hline
\end{tabular}

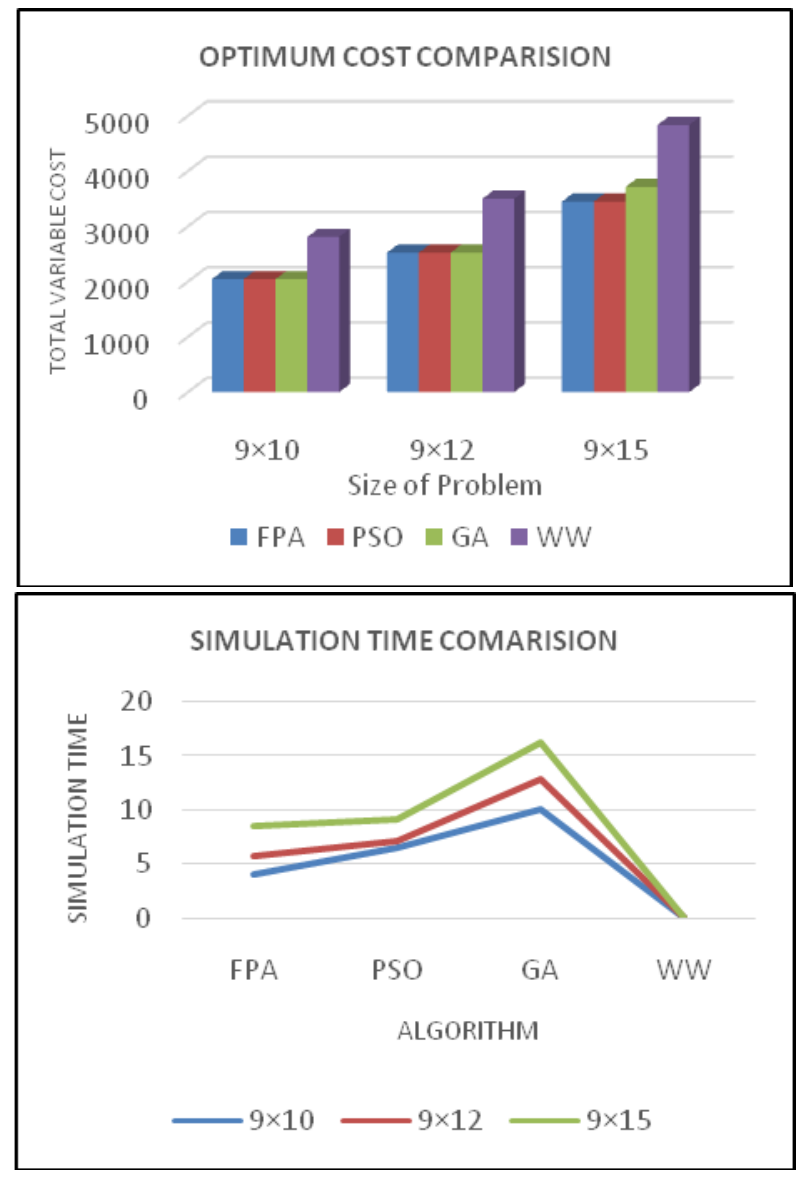

\section{Conclusion:}

Solving Multi level Lot sizing issues in manufacturing is one of the key issues of inventory control. The necessity of optimizing the cost in minimum time is very important in this era of rapidly changing environments. In these days of artificial intelligence and machine learning optimization of costs in very quick time is really an important concern.

Flower pollination algorithm was implemented for the inventory lot sizing problem with multiple levels

FPA algorithm outperformed all the other method solutions and successful in finding the optimum cost in reasonable amount of time.

It is efficient in terms of computational time aspect as well when compared with other evolutionary methods.
WW algorithm is quicker than FPA because it is a single pass method which is failed in finding the optimum solution.

\section{References:}

[1]. N. Dellaert, J. Jeunet, and N. Jonard, "Genetic algorithm to solve the general multi-level lot-sizing problem with time-varying costs," Int. J. Prod. Econ., vol. 68, no. 3, pp. 241-257, 2000, doi: 10.1016/S0925-5273(00)00084-0.

[2]. J. Jeunet and N. Jonard, "Single-point stochastic search algorithms for the multi-level lot-sizing problem," Comput. Oper. Res., vol. 32, no. 4, pp. 985-1006, 2005, doi: 10.1016/j.cor.2003.09.009. 
[3]. R. Kuik and M. Salomon, "Multi-level lot-sizing problem: Evaluation of a simulated-annealing heuristic,” Eur. J. Oper. Res., vol. 45, no. 1, pp. 2537, 1990, doi: 10.1016/0377-2217(90)90153-3.

[4]. W. Hernandez and G. A. Suer, "Genetic algorithms in lot sizing decisions," Proc. 1999 Congr. Evol. Comput. CEC 1999, vol. 3, no. 1, pp. 2280-2286, 1999, doi: 10.1109/CEC.1999.785558.

[5]. N. P. Dellaert and J. Jeunet, "Randomized multilevel lot-sizing heuristics for general product structures," Eur. J. Oper. Res., vol. 148, no. 1, pp. 211-228, 2003, doi: 10.1016/S03772217(02)00403-4.

[6]. F. Sahling, L. Buschkühl, H. Tempelmeier, and S. Helber, "Solving a multi-level capacitated lot sizing problem with multi-period setup carry-over via a fix-and-optimize heuristic," Comput. Oper. Res., vol. 36, no. 9, pp. 2546-2553, 2009, doi: 10.1016/j.cor.2008.10.009.

[7]. K. Wajanawichakon and R. Pitakaso, "Solving large unconstrainted multi level lot-sizing problem by a binary particle swarm optimization," Int. J. Manag. Sci. Eng. Manag., vol. 6, no. 2, pp. 133140, 2011, doi: 10.1080/17509653.2011.10671156.

[8]. H. M. Wagner and T. M. Whitin, "Dynamic version of the economic lot size model," Manage. Sci., vol. 50, no. 12 SUPPL., pp. 1770-1777, 2004, doi: 10.1287/mnsc. 1040.0262 .

[9]. S. K. Mishra, V. V.D. Sahithi, and C. S. P. Rao, “A hybrid binary particle swarm optimization for large capacitated multi item multi level lot sizing (CMIMLLS) problem," IOP Conf. Ser. Mater. Sci. Eng., vol. 149, no. 1, 2016, doi: 10.1088/1757899X/149/1/012040.

[10]. Mishra.S.K , Sahithi, V.V.D , Rao. C. (2016). A hybrid binary particle swarm optimization for large capacitated multi itemmulti level lot sizing (CMIMLLS) problem. IOP Conference Series: Materials Science and Engineering. 149. 012040. 10.1088/1757-899X/149/1/012040.

[11]. C. Almeder, "A hybrid optimization approach for multi-level capacitated lot-sizing problems," Eur. J. Oper. Res., vol. 200, no. 2, pp. 599-606, 2010, doi: 10.1016/j.ejor.2009.01.019.

[12]. Seyed Ashkan Hoseini Shekarabi, AbolfazlGharaei\& Mostafa Karimi (2019) Modelling and optimal lot-sizing of integrated multi-level multi-wholesaler supply chains under the shortage and limited warehouse space: generalised outer approximation, International Journal of Systems Science: Operations \& Logistics, 6:3, 237257, DOI: $10.1080 / 23302674.2018 .1435835$
[13]. Sahithi V.V.D., Rao C.S.P., Srinivasa Rao M. (2020) An Extensive Study of Multi-level Inventory Lot Sizing Optimization Problem. In: Voruganti H., Kumar K., Krishna P., Jin X. (eds) Advances in Applied Mechanical Engineering. Lecture Notes in Mechanical Engineering. Springer, Singapore. https://doi.org/10.1007/978-981-15-12018_126

[14]. P. Afentakis, B. Gavish, V. Karmarkar, Computationally efficient optimal solutions to the lot-sizing problem in multi-stage assembly systems, Management Science 30 (1984) 222-239.

[15]. H. Tempelmeier, M. Derstroff, A lagrangean-based heuristic for dynamic multilevel multiitem constrained lotsizing with setup times, Management Science 42 (1996) 738-757.

[16]. R. Pitakaso, C. Almeder, K.F. Doerner, R.F. Hartl, A MAX-MIN ant system for unconstrained multilevel lot-sizing problems, Computers and Operations Research 34 (2007) 2533-2552.

[17]. Woo Z, Hoon J, Loganathan GV. A New Heuristic Optimization Algorithm: Harmony Search. SIMULATION. 2001;76(2):60-68. doi:10.1177/003754970107600201

[18]. Xiao, Y.; Kaku, I.; Zhao, Q.; Zhang, R. A variable neighborhood search based approach for uncapacitated multilevel lot-sizing problems. Comput. Ind. Eng. 2011, 60, 218-227.

[19]. S. Pant, A. Kumar, and M. Ram, "Flower pollination algorithm development: a state of art review," Int. J. Syst. Assur. Eng. Manag., vol. 8, no. s2, pp. 1858-1866, 2017, doi: 10.1007/s13198-0170623-7.

[20]. Yi Han, Jiafu Tang, Iko Kaku, LifengMu,Solvinguncapacitated multilevel lotsizing problems using a particle swarm optimization with flexible inertial weight,Computers\& Mathematics with Applications,Volume 57, Issues 11-12,2009,Pages 1748-1755,ISSN 08981221,https://doi.org/10.1016/j.camwa.2008.10.024.

[21]. J. Xie, J.F. Dong, Heuristic genetic algorithm for general capacitated lot-sizing problems, Computer and Mathematics with Applications 44 (2002) 263276. 